\title{
Implantologie
}

\section{Neu: Bone Level Implantat mit konischem}

\section{Design}

Zum 35-jährigen Jubiläum präsentiert Straumann Deutschland ein neues Bone Level-Implantat mit konischem Design: Die konische Form ermöglicht eine Knochenkonduktion bei unterpräparierter Osteotomie und erleichtert Implantationen bei anatomischen Einschränkungen wie z. B. bei fazialem Unterschnitt. Das Implantat überzeugt durch die leistungs- starke Kombination aus neuem Design und bewährten klinischen Merkmalen der Straumann Bone Level Linie: Die Titan-Zirkonium-Legierung Roxolid und die hydrophile SLActive-Oberfläche ermöglichen eine besonders hohe Belastbarkeit und Osteokonduktivität; das Bone Control-Design, die CrossFit-Verbindung sowie die große prothetische Vielseitigkeit bieten weitere entscheidende Vorteile.

Nach einer Pressemitteilung der

Straumann GmbH, Freiburg 